\title{
Looking Back at 60 years of History to Pave a New Path for the Korean Society of Nuclear Medicine: Interviews with Senior Professors
}

\author{
Mijin Yun ${ }^{1} \cdot$ Hee-Seung Henry Bom ${ }^{2}$ (B) \\ Published online: 28 January 2022 \\ (C) The Author(s), under exclusive licence to Korean Society of Nuclear Medicine 2022
}

The year 2021 was the $60^{\text {th }}$ anniversary of the Korean Society of Nuclear Medicine (KSNM). In celebration, the Nuclear Medicine and Molecular Imaging (NMMI), the official journal of the KSNM, published review articles covering the development of various fields of nuclear medicine (NM) in South Korea in the issues of 2021. The editorial board (EB) members brainstormed ideas and discussed strategies to pave the way to a bright future for the NMMI. After numerous discussions, EB members decided to add artificial intelligence (AI) to the scopes and aims of the $N M M I$ and accordingly, the cover of the journal was changed, starting from this issue. The cover represents the application of AI to research and practice of NM. This year, a special issue will be published on the application of AI to NM. Any original articles, reviews, and letters from anyone interested in this topic are welcome.

In this issue, the editors interviewed two most senior professors, Prof. Yong Whee Bahk and Prof. Edmund Kim, who contributed to the foundation of the KSNM (Figure 1). We heard their life-long experiences which will guide us into the many future possibilities of NM. We hope to share this valuable knowledge with our readers and contribute to the continuous success of NM in South Korea.

\section{Interview with Prof. Yong Whee Bahk}

Springer Nature is a global publisher and publishes prominent NM journals such as the European Journal of Nuclear Medicine

Hee-Seung Henry Bom

henryhsbom@gmail.com

1 Department of Nuclear Medicine, Yonsei University College of Medicine, Seoul 03722, Republic of Korea

2 Department of Nuclear Medicine, Chonnam National University Medical School, Hwasun, Chonnam 58128, Republic of Korea and Molecular Imaging, Annals of Nuclear Medicine. Prof. Bahk had collaborated with Springer director Heilmann on several academic books. He shared his experience of approaching Springer for publication of his successful book on musculoskeletal pinhole imaging. He brought hundreds of x-rays and scanning films to show to the Springer director in Sweden. His book was agreed to be published after talks with the Springer New York headquarters. Due to the successful publication of his book, he gained the trust of Springer who decided to publish $N M M I$, raising the journal's status and to a global level.

Prof. Bahk reminded us of the history behind the Japanese Society of Nuclear Medicine (JSNM). As of now, the JSNM has five times more NM experts, resources, numerous published papers, and were far ahead in just about everything compared to the KSNM. Despite its smaller size, KSNM's strength is in imaging science and rightly so, imaging science using AI is a keyword for KSNM. The most important factor in NM is how well the image can represent concepts and theories of chemistry and physics. What's important is that we need to see it in order to understand pathophysiology. Being able to visually understand is the true meaning behind 'seeing is believing'. Prof. Bahk again emphasizes the quality of the image. The quality of the image should be comparable to that of in vivo immunohistochemistry and should be so clear that micrometric imaging diagnostics can be performed. In addition to theranostics, perfecting imaging science is one of the most important future goals for NM. German NM has the strength of being conservative but innovative and the USA is already a major market in NM. He strongly encourages us to collaborate and connect more with Germany and the USA.

Prof. Bahk has always preferred small handbooks over volumes of sheer size to concisely deliver powerful knowledge, similar to classics in Germany or France. NMMI can educate students by publishing journals, books, and editorials to emphasize that consistent work will eventually pay off. Above all, we need to pave the path for up and coming students so that they may jump into the challenging field of NM. 


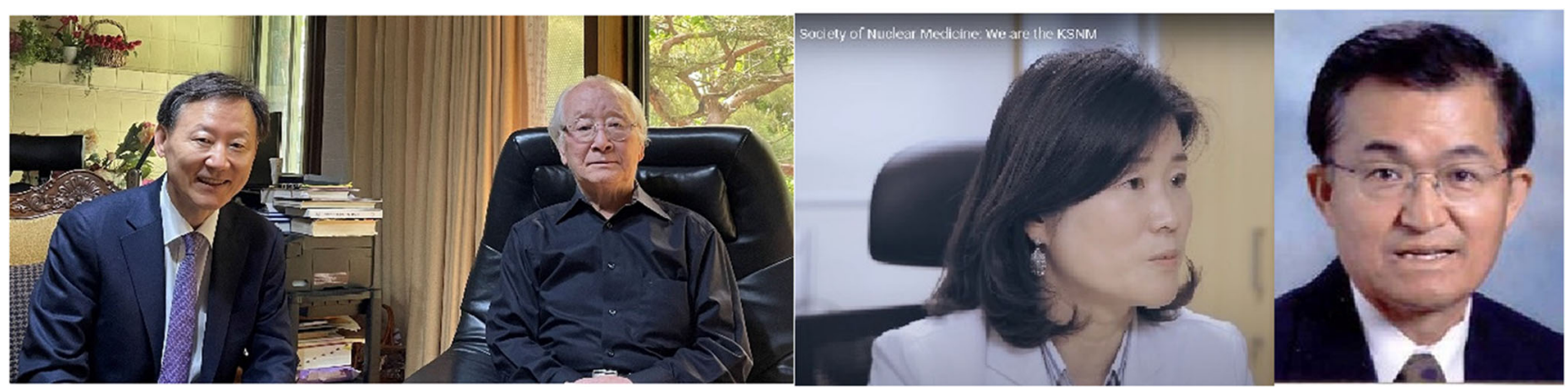

Figure 1 From left, Drs. Hee-Seung Henry Bom, Yong Whee Bahk, Mijin Yun, and Euishin Edmund Kim

\section{Interview with Prof. Euishin Edmund Kim}

Prof. Euishin Edmund Kim studied preventative medicine at the Seoul National University College of Medicine and internal medicine at the Johns Hopkins Hospital. When CT was first introduced, he became interested in radiology. In 1973, NM was officially established as a specialty. Dr. Kim worked as a faculty member in NM at the Washington University. Dr. Kim proposed to Dr. Goldenberg to radiolabel antibodies for radioimmunodetection and radioimmunotherapy. While he was working as an associate editor in Radiology, he had the chance to review a submission regarding nuclear magnetic resonance (NMR) imaging and became very interested in the subject. That led to him starting an international conference on NMR with Dr. Zang-hee Cho of the Columbia University, which turned out to be a huge start to magnetic resonance imaging. At MD Anderson, Dr. Kim practiced NM, thyroidology, and radiology. Recently doctors in the USA have started to prefer radiologists due to the influence of hybrid imaging. CT and MRI bring in the most profits in radiology, so there is little motivation to try out new things. NM has become a specialty that is replaceable by radiologists and now, the number in NM has become $1 / 10$ of radiologists in the USA.

Aside from interpreting images, NM can find answers to clinical questions and guide clinical practice. New radiopharmaceuticals targeting different aspects of disease would be of utmost importance to the future of NM. Theranostics should be further developed since it alone changed the world in the field of radioligand imaging and therapy. Radiogenomics, image-based personalized medicine, and microdosing all have good prospects for the future. Beyond the RNA level, radiometabolics and radioproteomics also have the potential to be developed further. All these future perspectives should meet unfulfilled clinical needs, which will push authorities such as the FDA to loosen regulations. NM presents limitless opportunities to learn something new through research. It is also a specialty that provides a service and requires communication with others. It can be classified into clinical service, research and education. NM in Korea has reached high standards in terms of journal publication, patient treatment, and education. Unfortunately, NM specialists in
Korea do not collaborate with other specialties so many innovations are not easily implemented into clinical practice due to lack of awareness.

He reminded us of the works of late Prof. Chang- Soon Koh, who played the role of an orchestra maestro, bringing various scientists and clinical specialists to the field of NM. It is important to not only understand the basis of NM and develop new techniques, but also to maintain ties with other people. In his dying wishes, late Prof. Koh said that we must help students cultivate their talents in order to promote the development of NM. It is extremely important that we cultivate interest and a sense of duty in the younger generation, as well as acquiring new knowledge from overseas conferences and applying it to clinical research and practice. It is a major issue if people with no interest and no motivation to work hard come into the field of NM. We need to have a positive influence on students, giving them praise and supporting their interests in order to show them the wonders of NM. Prof. Kim also mentioned that there seemed to be differences between east Asian philosophy and western values when it comes to educating students. Above all, the most important part of education is to consistently work hard and enjoy the process.

For the development of $N M M I$, Prof. Kim said that we need to carefully select and recruit reliable, world-renowned experts from other countries. Also, it is important to suggest a new direction for the development of NM through editorials, review articles...etc. Finally, by creating ties to the people at the publishing company, we also need to discuss publishing strategies to increase impact on biomedical societies, as well as public awareness of the NMMI.

\section{Declarations}

Conflict of interest Mijin Yun, Hee-Seung Henry Bom declare no conflict of interest.

Ethical statement No need of ethical approval. No need of informed consent 